\title{
COMPLEX NUMBERS, QUANTUM MECHANICS AND REALITY
}

\author{
Surmukh Singh Dhanoa ${ }^{1}$
}

\section{INTRODUCTION}

It is well known in mathematics that a complex number $\mathrm{z}$ is defined as $\mathrm{z}=\mathrm{x}+$ iy where both $\mathrm{x}$ and $\mathrm{y}$ are real and $\mathrm{i}=(-1)^{1 / 2}$. The quantities $\mathrm{x}$ and $\mathrm{y}$ are real and imaginary parts of $\mathrm{z}$ respectively. Rafael Bombelli, an Italian mathematician in sixteenth century invented complex number system and he himself regarded these as an useless invention since only real numbers corresponds to physical measureable quantities. However, later on many mathematicians formulated this system of numbers. The 'imaginary component' of complex numbers was introduced to accommodate the needs of mathematicians in order to manipulate the mathematical calculations. During the first quarter of twentieth century quantum mechanics was invented by Schrodinger to study the microscopic matter and presently it becomes a powerful tool in science and engineering to find the expected outcome of an experiment [1]. Recently in 2012 Higgs Boson also called 'God Particle' has been discovered experimentally [2,3] which was however predicted [4,5] through quantum mechanics in 1964. This branch of physics revolves around the complex number system. In fact, 'imaginary part' of complex number acts as 'converter' that converts imaginary quantity into real quantity. In quantum mechanics probability for the occurrence of an microscopic event is evaluated by taking into account the complex numbers, therefore complex numbers, quantum mechanics and reality are deeply interrelated with each other.

Imaginary number system can be compared with imagination of human mind since both keep no significance in reality. Human mind makes systematic efforts to convert imagination into reality. Quantum mechanics is the branch of physics that deals with invisible small particles called elementary particles [6]such as electron, proton, neutron, etc. and their mutual interactions. Quantum mechanics predicts the existence of such particles at a given place and time through complex calculations involving almost all branches of mathematics; and one such branch is the 'imaginary number system' which is the central player of all such calculations. Human mind imagined such predictions based upon quantum mechanics and then makes systematics efforts to convert such imaginations into reality. Remarkable discovery of $21^{\text {st }}$ century is the confirmation of existence of God particle through experiments $[2,3]$. The "God Particle" is the nickname of subatomic particle called the 'Higgs Boson' and it appears when head on collision between two protons moving at very high velocity occurs.

\footnotetext{
${ }^{1}$ Department of Mathematics, Akal Degree College, Mastuana Sahib, Sangrur, Punjab
} 
It is believed that mass of matter is due to the presence of God particle. Mass is the basic property of matter, that is why discovery of this particle is so remarkable.

\section{REFERENCES}

[1]. Kapustin, A. (2013). Is quantum mechanics exact? Journal of Mathematical Physics, 54, 062107.

[2]. CERN experiments observe particle consistent with long-sought Higgs Boson. CERN July 04, 2012.

[3]. The Higgs Boson. (2012). Scientific American ISBN: 978-1-466824133.

[4]. Higgs, P. (1964). Broken symmetry and the masses of gauge Bosons. Physical Review Letters . 13(6) 508-509.

[5]. Engrert, F. and Brout, R. (1964). Broken symmetry and the mass of gauge vector Meson. Physical Review Letters. 13(9) 321-323.

[6]. From the universe to the elementary particles: A first course introduction to cosmology and the fundamental interactions. Ulrich Ellwanger ISSN 2192-4791. Springer Heidelberg Dordrecht London. 